\title{
High resistivity and ultrafast carrier lifetime in argon implanted GaAs
}

\author{
W. Walukiewicz, ${ }^{\text {a) }}$ Z. Liliental-Weber, and J. Jasinski \\ Lawrence Berkeley National Laboratory, Berkeley, California 94720 \\ M. Almonte, A. Prasad, E. E. Haller, and E. R. Weber \\ Lawrence Berkeley National Laboratory and University of California, Berkeley, California 94720 \\ P. Grenier and J. F. Whitaker \\ Center for Ultrafast Optical Science, University of Michigan, Ann Arbor, Michigan 48109
}

(Received 24 June 1996; accepted for publication 15 August 1996)

\begin{abstract}
We have investigated the optoelectronic and structural properties of GaAs that has been implanted with Ar ions and subsequently annealed. The material exhibits all the basic optical and electronic characteristics typically observed in nonstoichiometric, As implanted or low-temperature-grown GaAs. Annealing of Ar implanted GaAs at $600{ }^{\circ} \mathrm{C}$ produces a highly resistive material with a subpicosecond trapping lifetime for photoexcited carriers. Transmission electron microscopy shows that, instead of As precipitates, characteristic for the nonstoichiometeric GaAs, voids ranging in size from 3 to $5 \mathrm{~nm}$ are observed in Ar implanted and annealed GaAs. (C) 1996 American Institute of Physics. [S0003-6951(96)03243-3]
\end{abstract}

Semiconductor materials grown at low temperatures have recently attracted considerable attention. It has been demonstrated that low temperature GaAs (LT GaAs) grown by molecular beam epitaxy (MBE) in the temperature range of $200-300{ }^{\circ} \mathrm{C}$ is highly nonstoichiometric with a large, up to $1 \%$, excess of As. ${ }^{1-4}$ In as-grown material the nonstoichiometry is accommodated by point defects: arsenic antisites, ${ }^{2}$ gallium vacancies ${ }^{5,6}$ and possibly by very small arsenic clusters. Upon annealing at about $600{ }^{\circ} \mathrm{C}$ most of the excess arsenic diffuses to form extended As precipitates. ${ }^{2,3}$ The appearance of the precipitates has been associated with a change in electronic properties, most notably with a large increase of the resistivity ${ }^{2,4,5}$ and shortening of the carrier lifetime. ${ }^{8,9}$ It has also been shown that similar properties can be achieved in As implanted and annealed GaAs. ${ }^{10,11}$

Although there is a consensus that precipitates and/or point defects play the key role in determining the properties of LT- and ion-implanted GaAs, the actual importance of different types of defects is still a controversial and hotly debated issue. ${ }^{2-5,7} \mathrm{~A}$ strong correlation between the presence of precipitates and the onset of desirable properties of LT- or ion-implanted $\mathrm{GaAs}$ has been established in numerous studies. ${ }^{3,12}$ Also, it has been reported that high resistivity GaAs can be produced by implantation of high doses of $\mathrm{Ni}$ and $\mathrm{Fe}$ which tend to precipitate upon high temperature annealing. ${ }^{13}$ The role of point defects is more difficult to elucidate, as they can be observed only indirectly and quite often their concentration is too low to be detected in thin implanted layers. Nevertheless, there is a body of evidence indicating that point defects are necessary if not sufficient for producing high resistivity, short carrier lifetime material. ${ }^{1,4,8}$ However, in all the previous studies the material was either highly nonstoichiometric or had high concentrations of metallic impurities. ${ }^{13}$ In both instances metallic precipitates were formed during the annealing. In order to determine if the metallic precipitates are necessary to achieve a high resistivity, short carrier lifetime material, we have performed

a)Electronic mail: wwladek@mh1.lbl.gov extensive investigations of the electrical and optical properties of stoichiometric GaAs implanted with inert argon gas ions. We expect this material to have a high concentration of damage induced native defects without metallic precipitates.

Semi-insulating (SI) and $n$-type GaAs wafers were implanted with Ar ions so that the depth of the damaged layer, as measured by Rutherford backscattering, was about 100 $\mathrm{nm}$. Samples were annealed for $30 \mathrm{~s}$ at temperatures $\left(T_{A}\right)$ between 300 and $950{ }^{\circ} \mathrm{C}$ using rapid thermal annealing (RTA). Room temperature Hall effect was measured on the implanted SI substrates and the $n$-type wafers were used for magnetic circular dichroism absorption (MCDA) measurements. Also low temperature sub-bandgap absorption was measured on the as-implanted and annealed samples.

In order to isolate the optical response of the implanted layer from that of the bulk the carrier lifetime measurements were performed on a MBE layer removed from its native substrate by epitaxial liftoff. The 400-nm-thick MBE layer was grown at $600{ }^{\circ} \mathrm{C}$. A proper implantation schedule with four different $\mathrm{Ar}$ ion doses and energies was employed to achieve uniform damage and full amorphization throughout the layer. Selected samples were studied by transmission electron microscopy (TEM) to reveal implantation induced extended defects.

Figures 1(a) and 1(b) show results of the Hall effect measurements for the samples implanted with Ar ions of energy $120 \mathrm{keV}$ and a dose of $1.6 \times 10^{16} \mathrm{~cm}^{-2}$. A relatively low sheet resistance and very low mobility in the as-implanted material can be attributed to a hopping conductivity via defects sites. ${ }^{2}$ The resistivity was found to increase with increasing annealing temperature. It reaches a maximum for an annealing temperature of about $600-700{ }^{\circ} \mathrm{C}$. A very significant increase in the electron mobility is also observed after annealing in this temperature range. The highest mobility of about $2000 \mathrm{~cm}^{2} / \mathrm{V} \mathrm{s}$ was found in a sample annealed at $700{ }^{\circ} \mathrm{C}$. At even higher annealing temperatures the mobility decreases again. A similar effect was previously observed by others and explained by a compensation with carbon acceptors adsorbed on chemically cleaned surfaces and incorpo- 

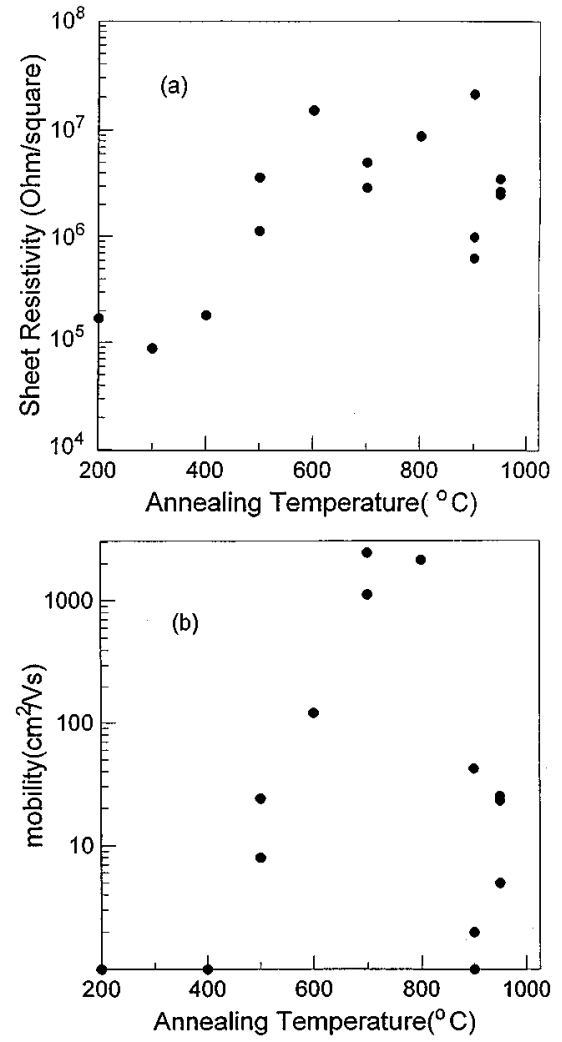

FIG. 1. Room temperature sheet resistivity (a) and carrier mobility (b) as functions of the annealing temperature.

rated through recoil implantation. ${ }^{14}$ The Hall effect results are consistent with infrared absorption measurements which show a very large reduction of the subband gap absorption upon annealing at $600{ }^{\circ} \mathrm{C}$ indicating a large decrease in the concentration of neutral $\mathrm{As}_{\mathrm{Ga}}$ (As antisites).

MCDA measurements were performed on $n$-type substrates implanted with the same Ar ion energy and dose. The MCDA signal can be calibrated to provide information on the concentration of singly ionized arsenic antisites. ${ }^{15}$ As shown in Fig. 2 an increase of the concentration $\mathrm{As}_{\mathrm{Ga}}^{+}$is observed for an annealing temperature $T_{A}$ starting somewhere between 350 and $400{ }^{\circ} \mathrm{C}$, and the maximum of $7 \times$ $10^{19} \mathrm{~cm}^{-3} \mathrm{As}_{\mathrm{Ga}}^{+}$was found for $T_{A}=500^{\circ} \mathrm{C}$. It is interesting

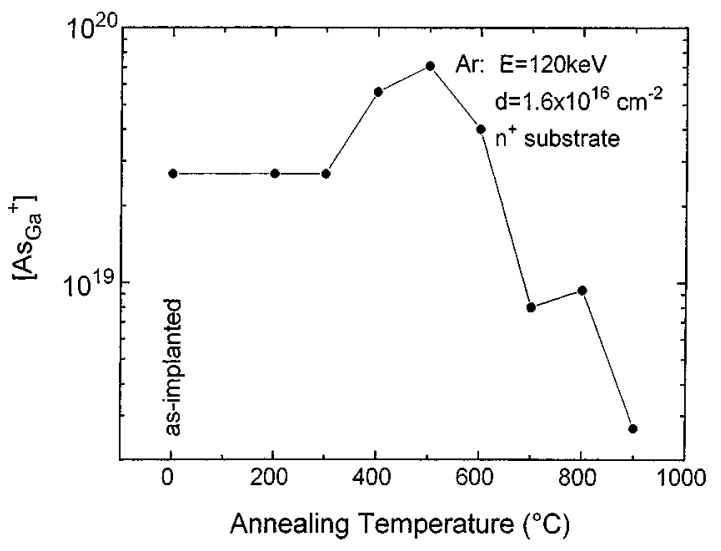

FIG. 2. The concentration of positively charged $\mathrm{As}_{\mathrm{Ga}}$ deduced from $\mathrm{MCD}$ absorption vs the annealing temperature.

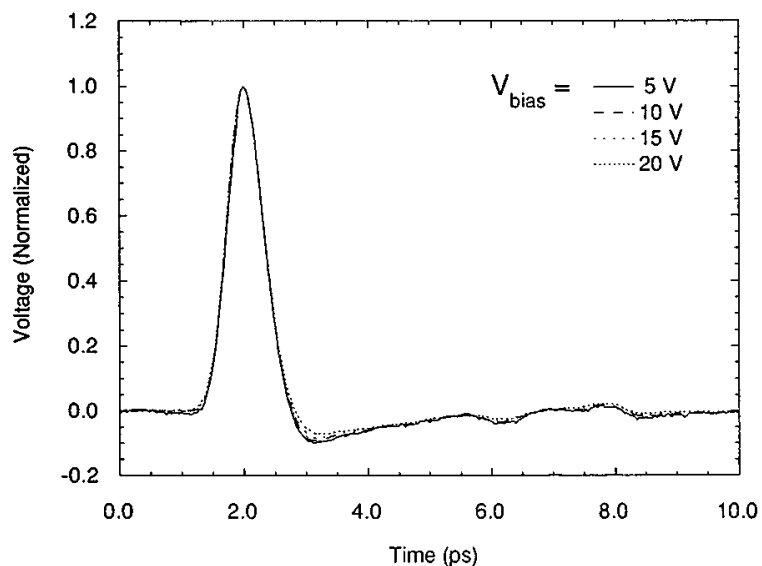

FIG. 3. Normalized output voltage pulses measured for several bias voltages on an $\mathrm{Ar}$ ion implanted GaAs detector.

to note that the maximum concentration of $\mathrm{As}_{\mathrm{Ga}}^{+}$corresponds to the annealing temperature where a rapid increase in the resistivity and mobility is found.

To further elucidate the properties of the Ar implanted GaAs we have measured the carrier lifetime. The measurements were performed on $a \approx 500$-nm-thick MBE grown GaAs layer implanted with Ar ions and lifted off the GaAs substrate. A $900 \mathrm{~nm} \mathrm{AlGaAs}$ buffer layer was used as an etch stop layer. In order to achieve a uniform damage profile the GaAs layer was consecutively implanted with $\mathrm{Ar}$ ions with energies of $275,125,80,50 \mathrm{keV}$ and doses of $3 \times 10^{15}, 9 \times$ $10^{14}, 1 \times 10^{14}, 4 \times 10^{14} \mathrm{~cm}^{-2}$, respectively. This resulted in a uniform distribution of implanted ions with a bulk concentration of about $1.6 \times 10^{20} \mathrm{~cm}^{-3}$. The material was annealed at $600{ }^{\circ} \mathrm{C}$, i.e., at the temperature where a maximum in electrical resistivity was observed. A bulk resistivity of the order of $10^{5} \Omega \mathrm{cm}$ was measured directly on the lifted off film.

Figure 3 shows normalized output voltage pulses obtained after applying several different bias voltages on a simple photoconductive detector fabricated on the GaAs layer. The $20-\mu \mathrm{m}$-wide photoconductive gap was illuminated by a $100 \mathrm{fs}$ laser pulse and the measurement was made using an external electro-optic sampling probe. The fast fall time indicates that the excited carriers are trapped within less than 1 ps. The return of the signal to the baseline, without any noticeable tail response, demonstrates that the trapping of photoexcited carriers is not only rapid but apparently also complete. The negative tail and the small bumps trailing the pulse are due to the response of the electro-optic probe and/or time domain reflections from the surrounding transmission line. The bias level of the photoconductor was varied in order to substantiate that there was no change in the signal versus electric field due to Schottky contacts or field screening. A similar reduction of the carrier trapping time was previously observed in proton implanted GaAs. ${ }^{16}$ It should be noted that one aspect of the ion implanted material that could be brought into questions, i.e., its reproducibility, was investigated through lifetime measurements on two different samples implanted at different times under identical conditions and annealed at $600{ }^{\circ} \mathrm{C}$. Results obtained using pump-probe femtosecond spectroscopy demonstrate that the 


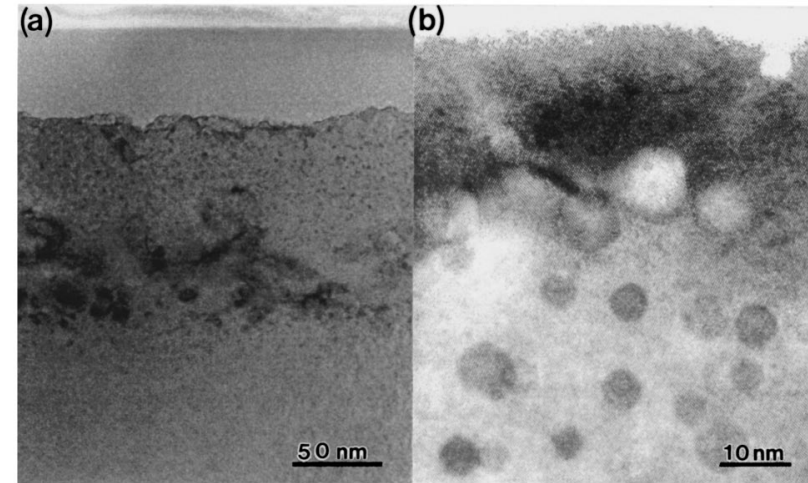

FIG. 4. Cross-sectional transmission electron micrographs of Ar ion implanted GaAs: (a) as implanted. Note the formation of an amorphous layer at the sample surface and the end of the range defects at about 100-160 nm below the surface. Small dots are the artifacts caused by the ion milling. (b) The same sample rapid thermal annealed at $950{ }^{\circ} \mathrm{C}$.

same, approximately $400 \mathrm{fs}$, trapping time is observed for both sets of samples.

The present study clearly shows that Ar implanted GaAs has all the essential optical and electronic properties of As implanted or LT grown GaAs. However, since in this case there is no excess As, we do not expect to have As precipitates. To confirm this presumption we have performed a TEM study of Ar implanted GaAs. We find that the as implanted sample has a 52-nm-thick amorphous layer at the sample surface [Fig. 4(a)]. We could not find any As precipitates in this material. After annealing at $600{ }^{\circ} \mathrm{C}$ voids with the diameters ranging from 3 to $5 \mathrm{~nm}$ become visible. As shown in Fig. 4(b), annealing at an even higher temperature of $950{ }^{\circ} \mathrm{C}$ leads to a coarsening of the voids and an increase of their diameter to $10 \mathrm{~nm}$. Although it is not obvious at the moment what role such voids play in determining the properties of this material, it is quite clear that unlike metallic precipitates the voids are not expected to accumulate a large excess charge and to form depleted regions.

In conclusion, our study shows that Ar implanted and annealed GaAs exhibit properties similar to those previously reported in As rich GaAs. We find that a high resistivity, high mobility, and short lifetime material can be obtained without metallic precipitates.

This work was supported by the Director, Office of Energy Research, Office of Basic Energy Sciences, Materials Sciences Division of the U.S. Department of Energy under Contract No. DE-AC03-76SF00098, by AFOSR-ISSA-900009 (LBNL), AFOSR under Grant No. DOD-6-F4930-951-0227 [University of Michigan (UM)], and by the NSF under Grant No. STC PHY 8920108 (UM).

${ }^{1}$ F. W. Smith, A. R. Calawa, C.-L. Chen, M. J. Mantra, and L. J. Mahoney, IEEE Electron. Device Lett. 9, 77 (1988).

${ }^{2}$ M. Kaminska, Z. Liliental-Weber, E. R. Weber, T. George, J. B. Kortright, F. W. Smith, B.-Y. Tsaur, and A. R. Calawa, Appl. Phys. Lett. 54, 1881 (1989); Z. Liliental-Weber, Mater. Res. Soc. Proc. 198, 371 (1991).

${ }^{3}$ A. C. Warren, J. M. Woodall, J. L. Freeouf, D. Grischkowsky, M. R. Melloch, and N. Otsuka, Appl. Phys. Lett. 57, 1331 (1990).

${ }^{4}$ D. C. Look, Thin Solid Films 231, 61 (1993).

${ }^{5}$ D. E. Bliss, W. Walukiewicz, J. W. Ager III, E. E. Haller, K. T. Chan, and S. Tanigawa, J. Appl. Phys. 71, 1699 (1992).

${ }^{6}$ S. M. McQuaid, R. C. Newman, M. Missous, and S. O'Hagan, Appl. Phys. Lett. 61, 3008 (1992).

${ }^{7}$ N. Atique, E. S. Harmon, J. C. P. Chang, J. M. Woodall, M. R. Melloch, and N. Otsuka, J. Appl. Phys. 77, 1471 (1994).

${ }^{8}$ S. Gupta, M. Y. Frankel, J. A. Valdmanis, J. F. Whitaker, F. W. Smith, and A. R. Calawa, Appl. Phys. Lett. 59, 3276 (1991).

${ }^{9}$ E. S. Harmon, M. R. Melloch, J. M. Woodall, D. D. Nolte, N. Otsuka, and C. L. Chang, Appl. Phys. Lett. 63, 2248 (1993).

${ }^{10}$ A. Claverie, F. Namavar, and Z. Liliental-Weber, Appl. Phys. Lett. 62, 1271 (1993).

${ }^{11}$ C. Jagadish, H. H. Tan, J. Jasinski, M. Kaminska, M. Palczewska, A. Krotkus, and S. Marcinkevicius, Appl. Phys. Lett. 67, 1724 (1995).

${ }^{12}$ See, for example, M. R. Melloch, J. M. Woodall, E. S. Harmon, N. Atique, D. D. Nolte, J. C. P. Chang, and N. Otsuka, Proceedings of the Semi-insulating III-V Materials Conference, Warsaw, Poland, 1994, edited by M. Godlewski (World Scientific, Singapore, 1994), p. 319, and references therein.

${ }^{13}$ J. C. P. Chang, N. Otsuka, E. S. Harmon, M. R. Melloch, and J. M. Woodall, Appl. Phys. Lett. 65, 2801 (1994).

${ }^{14}$ J. H. Makok and N. M. Haegel, Mater. Res. Symp. Proc. 240, 817 (1992).

${ }^{15}$ D. M. Hofmann, B. K. Mayer, J. M. Spaeth, M. Watlenbach, J. Krüger, C. Kisielowski-Kemmerich, and H. Alexander, J. Appl. Phys. 68, 3381 (1990).

${ }^{16}$ M. Lambsdorf, J. Kuhl, J. Rosenzweig, A. Axmann, and J. Schneider, Appl. Phys. Lett. 58, 1881 (1991). 